\title{
Kaon condensation at finite temperature
}

\author{
Toshitaka Tatsumi and Masatomi Yasuhira \\ Department of Physics, Kyoto University \\ Kyoto 606-01, Japan
}

\begin{abstract}
A new formulation is presented to treat thermal fluctuations around the kaon condensate, based on chiral symmetry. Separating the zero mode from the beginning we perform the imaginary-time path integral to the one loop to get the thermodynamic potential at finite temperature. The role of the Goldstone mode in the kaon condensed phase is elucidated in relation to the equation of state.
\end{abstract}

PACS: 11.30.Rd; 12.39.Fe; 21.65.+f; 26.60.+c

Keywords: Chiral symmetry; Kaon condensate; Thermal fluctuations; Equation of state; Protoneutron stars 
Kaon condensation has been extensively studied for years [1]. As their implications there have been suggested many phenomena in relation to neutron stars. Among them the lowmass black-hole scenario, proposed by Brown and Bethe [2], gives one of most interesting possibilities for explaining the current observation on the mass of neutron stars or the wouldbe neutron star in the SN1987A. According to this scenario a newly formed hot neutron star after the supernova explosion may collapse to a black hole (the delayed collapse) during the deleptonization or the initial cooling era in the star's first $\sim 20$ s due to the softening of the equation of state (EOS). A numerical simulation has been already done following this scenario by Baumgarte et al. [3]. Through the studies of EOS it has been found that kaon condensation results in the large softening of EOS and a low maximum mass for neutron stars. It may widely lie from $1.4 M_{\odot}$ to $2.1 M_{\odot}$ in a relativistic calculation by Fujii et al. 《四. A nonrelativistic calculation by Thorsson et al also suggested similar values [5]. Anyway kaon condensation gives a possibilty of a low maximum mass of $\sim 1.4 M_{\odot}$, and thereby the low mass black holes can be expected around $1.4 M_{\odot}$ [2].

EOS for the kaon condensed phase has been studied mostly for low temperature. However, the delayed collapse may occur in the hot protoneutron-star regime at temperature, $T \sim$ several tens of $\mathrm{MeV}$, as suggested in the simulations [3].6]. Hence the studies of kaon condensation at finite temperature is required. Since there was no EOS of the kaon condensate at finite temperature, Baumgarte et al. used EOS at $T \simeq 0$ in their numerical simulation [3]. Recently, Prakash et al. treated the kaon-condensed phase at finite temperature and discussed the properties of protoneutron stars within the meson exchange model, since there is no consistent theory based on chiral symmetry [7].

Chiral symmetry is an important concept for kaon condensation; the kaon condensed phase can be represented as a chirally-transformed state from the vacuum in terms of chiral symmetry, and the essential results can be obtained in a model-independent way [4]. So we wish a consistent treatment of the thermal fluctuations based on chiral symmetry even at finite temperature. In a recent paper Thorsson and Ellis have tried to include quantum or thermal fluctuations within a chiral theory [8]. However, they have discussed only the effect of the zero-point energy of kaons (quantum fluctuations). Furthermore their expression for the thermodynamic potential looks too complicated to be tractable.

In this Letter we present a new formalism to treat thermal fluctuations on the basis of chiral symmetry and demonstrate how our formalism works by calculating EOS. We shall see that our formalism makes the analysis of the structure about the kaon dynamics possible and clarifies the physics included in the condensed phase. We consider here the isothermal system without trapping of neutrinos for simplicity. We treat nucleons as a kind of background for kaons and do not take into account their dynamical degrees of freedom except the kinetic energy, which may follow the spirit by the heavy-baryon chiral-perturbation theory [9]. Relativistic effects for nucleons are beyond the scope of this Letter.

We start with the Kaplan-Nelson Lagrangian with the Goldstone field $U$ and the Baryon octet $B$ as an effective chiral Lagrangian $\mathcal{L}_{\text {chiral }}$ [1,10], which consists of the symmetric part,

$$
\mathcal{L}_{0}=\frac{f^{2}}{4} \operatorname{tr}\left(\partial_{\mu} U^{\dagger} \partial^{\mu} U\right)+i \operatorname{tr}\left\{\bar{B}\left(D-m_{B}\right) B\right\}+D \operatorname{tr}\left(\bar{B} \gamma_{\mu} \gamma_{5}\left\{A^{\mu}, B\right\}\right)+F \operatorname{tr}\left(\bar{B} \gamma_{\mu} \gamma_{5}\left[A^{\mu}, B\right]\right)
$$

and the breaking part, 


$$
\begin{aligned}
\mathcal{L}_{S B} & =\alpha \operatorname{tr} \hat{m}_{q}\left(U+U^{\dagger}-2\right) \\
& +a_{1} \operatorname{tr} \bar{B}\left(\xi \hat{m}_{q} \xi+\text { h.c. }\right) B+a_{2} \operatorname{tr} \bar{B} B\left(\xi \hat{m}_{q} \xi+\text { h.c. }\right)+a_{3}\{\operatorname{tr} \bar{B} B\} \operatorname{tr}\left(\hat{m}_{q} U+\text { h.c. }\right)
\end{aligned}
$$

with the quark mass-matrix, $\hat{m}_{q} \simeq \operatorname{diag}\left(0,0, m_{s}\right)$. The coefficients $\alpha, a_{i}$ measure the strength of the explicitly symmetry breaking: the kaon mass is given as $m_{K}^{2} \simeq 2 m_{s} \alpha / f^{2}$ and the $K N$ sigma terms as $\Sigma_{K p}=-m_{s}\left(a_{1}+a_{2}+2 a_{3}\right)$ and $\Sigma_{K n}=-m_{s}\left(a_{2}+2 a_{3}\right)$. The transformation properties of the fields $U$ and $B$ under $S U(3)_{L} \times S U(3)_{R}$ are found in ref. [10.

It is well-known that chemical potentials, which is needed to ensure various conservation laws in the ground state, can be introduced as the artificial "gauge fields". Here we must take into account the conservation laws of two quantities: electromagnetic charge and baryon number. Accordingly we replace the time-derivatives by the "covariant derivatives"; $\mathcal{D}_{\mu=0} U=\partial U / \partial t+i \mu_{K}\left[T_{e m}, U\right], \mathcal{D}_{\mu=0} B=\partial B / \partial t-i \mu_{n} B+i \mu_{K}\left[T_{e m}, B\right]$, with the chemical potentials $\mu_{K}, \mu_{n}$, and the charge operator, $T_{e m} \equiv T_{3}+1 / \sqrt{3} T_{8}=\operatorname{diag}(2 / 3,-1 / 3,-1 / 3)$ $\left(T_{a}\right.$ : generators of $\left.S U(3)\right)$. Thus the partition function in the imaginary-time formalism can be represented as follows $(\tau=i t, \beta=1 / T)$;

$$
Z_{\text {chiral }}=N \int[d U][d B][d \bar{B}] \exp \left[\int_{0}^{\beta} d \tau \int d^{3} x\left(\mathcal{L}_{\text {chiral }}+\delta \mathcal{L}\right)\right] .
$$

Here we can see that there appears the additional term in the effective action, $\delta \mathcal{L}$, which is in general non-invariant under the chiral transformation,

$$
\begin{aligned}
\delta \mathcal{L} & =-\frac{f^{2} \mu_{K}}{4} \operatorname{tr}\left\{\left[T_{e m}, U\right] \dot{U}^{\dagger}+\dot{U}\left[T_{e m}, U^{\dagger}\right]\right\}-\frac{\mu_{K}}{2} \operatorname{tr}\left\{B^{\dagger}\left[\left(\xi^{\dagger}\left[T_{e m}, \xi\right]+\xi\left[T_{e m}, \xi^{\dagger}\right]\right), B\right]\right\} \\
& -\frac{f^{2} \mu_{K}^{2}}{4} \operatorname{tr}\left\{\left[T_{e m}, U\right]\left[T_{e m}, U^{\dagger}\right]\right\}+\mu_{n} \operatorname{tr}\left\{B^{\dagger} B\right\}-\mu_{K} \operatorname{tr}\left\{B^{\dagger}\left[T_{e m}, B\right]\right\},
\end{aligned}
$$

where $\dot{U} \equiv \partial U / \partial \tau$.

Fluctuations around the condensate are introduced as follows. First consider the chiral transformations on the $S U(3)_{L} \times S U(3)_{R} / S U(3)_{V} \simeq S U(3)$ manifold. Then the eight Goldstone fields $\phi_{a}$ span the coordinates on the manifold [11], $U\left(\phi_{a}\right)=\exp \left(2 i T_{a} \phi_{a} / f\right) \in S U(3)$, and accordingly the kaon condensed phase corresponds to the point on the manifold specified by $U_{K}=\exp \left[2 i\left\{T_{4}\left\langle\phi_{4}\right\rangle+T_{5}\left\langle\phi_{5}\right\rangle\right\} / f\right]$. I On the other hand we know that it is attained by the chiral transformation from the vacuum, $U_{V}=U\left(\phi_{a}=0\right)=1$,

$$
U_{K}(\langle\theta\rangle)=\zeta U_{V} \zeta=\zeta^{2}
$$

where $\zeta=\exp (i\langle M\rangle / \sqrt{2} f)$ with the kaon matrix

$M=\left[\begin{array}{ccc}0 & 0 & K^{+} \\ 0 & 0 & 0 \\ K^{-} & 0 & 0\end{array}\right], \quad K^{ \pm}=\left(\phi_{4} \pm i \phi_{5}\right) / \sqrt{2} \quad$ and $\quad \theta^{2} \equiv 2 K^{+} K^{-} / f^{2}$

Thorsson and Ellis introduced the kaon fluctuation fields $\tilde{K}^{ \pm}$in the usual manner [8], $K^{ \pm}=\left\langle K^{ \pm}\right\rangle+\tilde{K}^{ \pm}$. Here we introduce the fluctuation fields by extending the idea of chiral

\footnotetext{
${ }^{1}$ The symbol $\langle\mathcal{O}\rangle$ implies the thermal average of an operator $\mathcal{O}$.
} 
rotation mentioned above. First, we generate fluctuations $\phi_{a}$ around the vacuum by a chiral transformation $U_{f}, U_{f}=\eta U_{V} \eta=\eta^{2}$ with $\eta=\exp \left(i T_{a} \phi_{a} / f\right)$. Then an additional chiral rotation by $\zeta$ transforms $U_{f}$ into the form,

$$
U\left(\langle\theta\rangle, \phi_{a}\right)=\zeta U_{f}\left(\phi_{a}\right) \zeta
$$

This is our ansatz for the form of $U$ on the manifold. It is obvious that in the limit $\phi_{a} \rightarrow 0$ or $\langle\theta\rangle \rightarrow 0$, the matrix $U$ is reduced to the relevant form. We can regard this procedure as a separation of the "zero-mode" from the full $S U(3)$ matrix $U$ [12,14. Accordingly, the $\xi$ operator, which is defined by $U=\xi^{2}$, can be obtained by solving the subsidiary equation,

$$
\xi=\zeta \eta u^{\dagger}=u \eta \zeta
$$

where the matrix $u$ is defined by the second equality in Eq.(7).

Defining a new baryon field $B^{\prime}$ by the use of the matrix $u, B^{\prime}=u^{\dagger} B u$, we can see that the chiral-invariant pieces of the Lagrangian are not changed in form and only the symmetry breaking pieces play crucial roles,

$$
\begin{aligned}
\mathcal{L}(U, B)=\mathcal{L}_{0}(U, B)+\mathcal{L}_{S B}(U, B) & \longrightarrow \mathcal{L}_{0}\left(U_{f}, B^{\prime}\right)+\mathcal{L}_{S B}\left(\zeta U_{f} \zeta, u B^{\prime} u^{\dagger}\right) \\
\delta \mathcal{L}(U, B) & \longrightarrow \delta \mathcal{L}\left(\zeta U_{f} \zeta, u B^{\prime} u^{\dagger}\right) .
\end{aligned}
$$

Thus all the dynamics among the condensate, fluctuations and baryons are completely prescribed by the non-invariant terms under the chiral transformation, $\mathcal{L}_{S B}$ and $\delta \mathcal{L}$.

To proceed we have to find the $S U(3)$ matrix $u$ by Eq.(17). It depends on $\phi_{a},\langle\theta\rangle$ in a complicated nonlinear way, and thereby it is very difficult to find $u$ for the general form of $U_{f}$ [10]. However, if we restrict ourselves to the fluctuations of kaon sector s.t.

$$
U_{f}=\exp (i \sqrt{2} M / f)
$$

then we can simply find

$$
u=\operatorname{diag}\left(\kappa^{*} /|\kappa|, 1, \kappa /|\kappa|\right)
$$

with

$$
\kappa=\cos (\langle\theta\rangle / 2) \cos (\theta / 2)-\frac{K^{+}\left\langle K^{-}\right\rangle}{\left|\left\langle K^{-}\right\rangle\right|\left|K^{+}\right|} \sin (\langle\theta\rangle / 2) \sin (\theta / 2) .
$$

For the integration measure in Eq.(3), it is invariant under the transformation (6) by $\zeta$, $[d U] \rightarrow\left[d U_{f}\right]$, and further approximated by the one with flat curvature [12]. Thus we find

$$
Z_{\text {chiral }} \simeq \int \prod_{i=1}^{8}\left[d \phi_{i}\right]\left[d B^{\prime}\right]\left[d \bar{B}^{\prime}\right] \exp \left[S_{\text {chiral }}^{\text {eff }}\left(\zeta, U_{f}, B^{\prime}\right)\right]
$$

In the following calculation we only consider the kaon dynamics in nuclear matter $(B \rightarrow$ $\psi)$ in the one-loop order. As mentioned before, since we discard the dynamical degrees of freedom of nucleons in this Letter, we can integrate out the kaon field and the nucleon field separately in Eq. (12). First we consider the integration over the kaon field. Using Eq. (10) 
and retaining only the quadratic terms with respect to the kaon field in Eq. (8), we can write the effective action as

$$
S_{\text {chiral }}^{e f f}=S_{c}+S_{K}^{e f f}
$$

apart from the nucleon terms, with the tree-level contribution,

$$
S_{c}=\beta V\left[\frac{1}{2} \mu_{K}^{2} f^{2} \sin ^{2}\langle\theta\rangle-f^{2}\left(m_{K}^{2}-\sigma-2 \mu_{K} b\right)(1-\cos \langle\theta\rangle)\right]
$$

and the fluctuation part,

$$
\begin{aligned}
S_{K}^{e f f}=\int_{0}^{\beta} d \tau \int d^{3} x & {\left[-\left(\dot{K}^{-}-\mu_{K} \cos \langle\theta\rangle K^{-}\right)\left(\dot{K}^{+}+\mu_{K} \cos \langle\theta\rangle K^{+}\right)-\nabla K^{+} \nabla K^{-}\right.} \\
& -\cos \langle\theta\rangle\left(m_{K}^{2}-\sigma\right) K^{+} K^{-}-\frac{\mu_{K}^{2}}{4} \sin ^{2}\langle\theta\rangle\left(K^{+}+K^{-}\right)^{2} \\
- & \left.b\left\{K^{+}\left(\dot{K}^{-}-\mu_{K} \cos \langle\theta\rangle K^{-}\right)-K^{-}\left(\dot{K}^{+}+\mu_{K} \cos \langle\theta\rangle K^{+}\right)\right\}+O\left(|K|^{4}\right)\right],
\end{aligned}
$$

where $b$ stands for the $\mathrm{V}$-spin density, $b=\left\langle\bar{\psi} \gamma_{0} \frac{1}{8}\left(3+\tau_{3}\right) \psi\right\rangle / f^{2}$ and $\sigma$ the $K N$ sigma-term contribution, $\sigma=\left\langle\bar{\psi} \Sigma_{K N} \psi\right\rangle / f^{2}$ with $\Sigma_{K N}=\left(\begin{array}{cc}\Sigma_{K p} & 0 \\ 0 & \Sigma_{K n}\end{array}\right)$. U Note that Eqs. (14),

are the exact consequences from the general argument given in Eq. (8). Expanding the fluctuation field with the Matsubara frequency $\omega_{n}=2 \pi n T$ and performing the integration, we find the kaon-loop contribution,

$$
\ln Z_{K}^{e f f}=\sum_{n, \mathbf{p}} \ln \left(\left|D^{e f f}\right|^{-1 / 2}\right)
$$

with the inverse thermal Green function $D^{e f f}$,

$$
D^{e f f}=\beta^{2}\left(\begin{array}{cc}
\omega_{n}^{2}+\left(\tilde{\omega}_{-}-\tilde{\mu}_{K}\right)\left(\tilde{\omega}_{+}+\tilde{\mu}_{K}\right)+\mu_{K}^{2} \sin ^{2}\langle\theta\rangle & 2\left(\tilde{\mu}_{K}+b\right) \omega_{n} \\
-2\left(\tilde{\mu}_{K}+b\right) \omega_{n} & \omega_{n}^{2}+\left(\tilde{\omega}_{-}-\tilde{\mu}_{K}\right)\left(\tilde{\omega}_{+}+\tilde{\mu}_{K}\right)
\end{array}\right),
$$

where $\tilde{\omega}_{ \pm}= \pm b+\left(p^{2}+\tilde{m}_{K}^{* 2}+b^{2}\right)^{1 / 2}, \tilde{\mu}_{K}=\mu_{K} \cos \langle\theta\rangle$ and the effective mass, $\tilde{m}_{K}^{* 2}=\cos \langle\theta\rangle\left[m_{K}^{2}-\right.$ $\sigma]$. p

The excitation energy of the kaonic modes is given by the poles of the thermal Green function. Then we have two solutions corresponding to the $K^{ \pm}$modes,

$$
E_{ \pm}^{2}=\left(c_{1}+c_{3}+2 c_{2}^{2}\right) \pm \sqrt{\left(c_{3}+2 c_{2}^{2}\right)^{2}+4 c_{1} c_{2}^{2}}
$$

\footnotetext{
${ }^{2}$ It would be interesting to compare our result with the one given in ref. [8]; although our expression might look rather simple, the results, e.g. the dispersion relations for the kaonic modes, are consistent with them.

${ }^{3}$ In the limit $\langle\theta\rangle \rightarrow 0, \tilde{\omega}_{ \pm} \rightarrow \omega_{ \pm}= \pm b+\left(p^{2}+m_{K}^{* 2}+b^{2}\right)^{1 / 2}$, which is nothing but the dispersion relation for kaons in the normal phase [1]:何.
} 
where $c_{1}=\left(\tilde{\omega}_{-}-\tilde{\mu}_{K}\right)\left(\tilde{\omega}_{+}+\tilde{\mu}_{K}\right), c_{2}=\tilde{\mu}_{K}+b$ and $c_{3}=1 / 2 \mu_{K}^{2} \sin ^{2}\langle\theta\rangle$. In the condensed phase $(\langle\theta\rangle \neq 0)$, the mode corresponding to $E_{-}$is the Goldstone mode as a consequence of the breakdown of $\mathrm{V}$-spin symmerty in the ground state; the ground state is not an eigenstate of the conserved operator $V_{3}$. It is easy to show $E_{-}(\mathbf{p}=0) \sim 0$. In the tree-level approximation, $c_{1}=0$ as a result of the extremum condition for $S_{c}, \partial S_{c} / \partial\langle\theta\rangle=0$, which means $E_{-}=0$, while we should find $E_{-}(\mathbf{p}=0)=0+O(\hbar)$ once taking into account the fluctuation effects (kaon loops) [8]. When we consider the thermal kaon loops, $E_{-}$directly enters into the Bose-Einstein distribution function and it should diverge at $\mathbf{p}=0$. The other is the massive mode and can be neglected for temperature we are interested in $(T \leq 100 \mathrm{MeV})$.

Moreover, if we can safely neglect the $c_{3}$ term, we get a simple expression for $E_{ \pm}$,

$$
E_{ \pm}=\tilde{\omega}_{ \pm} \pm \tilde{\mu}_{K}=\sqrt{p^{2}+\tilde{m}_{K}^{* 2}+b^{2}} \pm\left(b+\tilde{\mu}_{K}\right) .
$$

We can expect the pertinence of this approximated formula qualitatively by several reasons: first, this term includes the $K K$ scattering amplitude in the leading order in difference from the other terms in Eq. (17), which should be small at low-energy regime due to the Goldstone-particle nature of kaons. Secondly, in the limit, $\langle\theta\rangle \rightarrow 0$ or $\mu_{K} \rightarrow 0, c_{3}$ gives no contribution. We also know that $\langle\theta\rangle$ and $\mu_{K}$ are inversely proportional to each other referring the previous studies, e.g., [1,4.5]. Leaving the detailed discussions in ref. [14], we find that the approximation is justified if the following condition is satisfied,

$$
c_{3} \ll \frac{C \pi}{16} T \zeta^{2}(3 / 2) \quad \text { or } \quad \mu_{K} \sin \langle\theta\rangle \ll \frac{1}{4}(2 \pi C T)^{1 / 2} \zeta(3 / 2),
$$

with $C^{2}=\tilde{m}_{K}^{* 2}+b^{2}$. We shall see, by numerical calculations, that this approximation really works well even at finite temperature except very low temperature, where thermal effects should be trivially unimportant. Finally, it is to be noted that this approximation never violate the Goldstone-particle nature, $E_{-}(\mathbf{p}=0)=0+O(\hbar)$.

Then the thermodynamic potential by the kaon-loop contribution, $\Omega_{K}^{e f f}=-T \ln Z_{K}^{e f f}$, reads

$$
\Omega_{K}^{e f f}=V \int \frac{d^{3} p}{(2 \pi)^{3}} \frac{1}{2}\left[E_{+}(\mathbf{p})+E_{-}(\mathbf{p})\right]+T V \int \frac{d^{3} p}{(2 \pi)^{3}} \ln \left(1-e^{-\beta E_{+}(\mathbf{p})}\right)\left(1-e^{-\beta E_{-}(\mathbf{p})}\right) .
$$

The first term is the zero-point energy (ZP) contribution and the second one the thermal one. Since ZP contribution includes the divergent integrals, they should be renormalized in some way. Following the method proposed in ref. [8], we can extract the finite contribution $\Omega^{Z P, r} ;$ under the approximation, $c_{3}=0, \Omega^{Z P, r}$ takes a simple form,

$$
\Omega^{Z P, r} \sim-\frac{1}{64 \pi^{2}} V\left[\left(m_{K}^{2}-3 C^{2}\right)\left(m_{K}^{2}-C^{2}\right)+2 C^{4} \ln \frac{m_{K}^{2}}{C^{2}}\right] .
$$

We shall see numerically that $\Omega^{Z P, r}$ results in a tiny value, as consistent with ref. [8], we discard it in the following.

In the rest of this Letter we present some results about the dispersion relation and EOS at finite temperature. For nucleons we should take into account at least two effects, otherwise the results should become meaningless. First one is the thermal effects for the kinetic energy term; it is given by the standard formula, 


$$
\Omega_{N}^{k i n} \simeq-2 T V \sum_{n, p} \int \frac{d^{3} p}{(2 \pi)^{3}} \ln \left(1+e^{-\beta\left(\epsilon-\mu_{i}\right)}\right)
$$

where $\epsilon=p^{2} / 2 M$ for nonrelativistic nucleons and $\mu_{p}$ is defined by the relation, $\mu_{p}=\mu_{n}-\mu_{K}$. Secondly, it is well-known that the nuclear symmetry energy plays an important role for the ground-state properties of the condensed phase. Since we have already included the kinetic energy for nucleons, we take into account only the potential energy contribution. Following Prakash et al. [13] we effectively introduce a symmetry energy contribution,

$$
\Omega_{N}^{\text {symm }}=V \rho_{B}(1-2 x)^{2} S^{p o t}(u),
$$

where $\rho_{B}$ the baryon number density, $u=\rho_{B} / \rho_{0}\left(\rho_{0} \simeq 0.16 \mathrm{fm}^{-3}\right.$ the nuclear density), and $x$ is the proton-number fraction. The coefficient $S^{\text {pot }}(u)$ is given as $S^{\text {pot }}(u)=\left(S_{0}-\left(2^{2 / 3}-\right.\right.$ 1) $\left.(3 / 5) E_{F}^{0}\right) F(u)$ with the empirical symmetry energy $S_{0} \simeq 30 \mathrm{MeV}$ and the Fermi energy $E_{F}^{0}$ at $\rho_{0}$. We take here $F(u)=u$ as a form of the control function for simplicity. Thus the nucleon contribution is given by $\Omega_{N}^{e f f}=\Omega_{N}^{k i n}+\Omega_{N}^{s y m m}$.

Finally, the total thermodynamic potential $\Omega_{\text {total }}$ is given by further adding the one for leptons (electrons and muons), $\Omega_{l}$,

$$
\Omega_{\text {total }}=\Omega_{c}+\Omega_{K}^{e f f}+\Omega_{N}^{e f f}+\Omega_{l},
$$

where $\Omega_{c}$ is the tree-level contribution, $\Omega_{c}=-T S_{c}$, given by Eq. (14) and we can use the formulae of free leptons for $\Omega_{l}$. The parameters $x,\langle\theta\rangle$ and the chemical potential $\mu_{K}$ are determined by the extremum conditions for given density and temperature like in the $T=0$ case, e.g., [1,4,5]: $\partial \Omega_{\text {total }} / \partial x=0, \partial \Omega_{\text {total }} / \partial\langle\theta\rangle=0$ and $\partial \Omega_{\text {total }} / \partial \mu_{K}=0$ by the use of Eq.(25).

In the following we present some numerical examples to see how our formalism works and how large the thermal effects are, leaving the full discussion about the thermal effects and the parameter dependence in a separated paper [14]. In numerical calculations we use the values, $a_{1} m_{s}=-67 \mathrm{MeV}, a_{2} m_{s}=134 \mathrm{MeV}$ and $a_{3} m_{s}=-222 \mathrm{MeV}$ [5]. First of all we examine the pertinence of the approximation $c_{3}=0$. We compared the exact dispersion relation (18) with the approximated one (19) as a function of momentum up to $500 \mathrm{MeV}$ and checked that this is a fairly good approximation. In Fig.1 we show a typical example, which might be the worst case at $T=50 \mathrm{MeV}$ in such a sense that the difference between them becomes largest. We can see that the difference is very small; in fact, it causes the error of only less than several $\mathrm{MeV} \cdot \mathrm{fm}^{-3}$ for pressure. As a general trend the difference becomes smaller as density or temperature becomes higher (c.f. Eq. (20) ). It should be worth noting that once this approximation works, the expressions of thermodynamic quantities like charge, entropy, energy become very clear [14].

In Fig. 2 we present an example of EOS for the hot neutron-star matter by using $c_{3}=0$. We construct EOS for the condensed phase, referring to the one for normal neutron-star matter; $P=P_{V}+P_{\text {chiral }}$. The pressure $P_{\text {chiral }}$ is simply given by Eq.(25) through the

\footnotetext{
${ }^{4}$ We also compared our dispersion relations with ref. [8] at $T=0$ and found that they are consistent with each other regardless of the magnitude of $\langle\theta\rangle$ in the ground state.
} 
thermodynamic relation, $P_{\text {chiral }} V=-\Omega_{\text {tot }}$. As a reference EOS for normal neutron-star matter we employ here the one suggested by Prakash et al. [13]; $P_{V}=u^{2} \partial(V(u) / u) / \partial u$ with the potential contribution $V(u)$. The results indicate the first-order phase transition in Fig. 2. Hence, the Maxwell construction should be imposed to get the real EOS. We can easily see that the thermal effects are remarkably larger than those in the normal phase; this is caused by the reduction of the chiral angle, and the soft mode mainly contributes to this effect.

Finally we have checked the smallness of the ZP contribution to be tiny; the value of the ZP contribution to pressure, $P_{Z P}=-\Omega^{Z P, r} / V$, is always less than several $\mathrm{MeV} \cdot \mathrm{fm}^{-3}$ over the density-temperature region we are interested in.

In this Letter we have presented a formalism to treat fluctuations around the condensate within the framework of chiral symmetry. Our approach is based on the group theoretical argument; Goldstone fields are regarded as the coordinates on the chiral $S U(3)_{L} \times S U(3)_{R} / S U(3)_{V}$ manifold and the kaon condensed phase can be represented as a chiral rotated state from the vacuum. Using this idea we have introduced fluctuations around the condensate by way of the successive chiral transformations. We derived an effective action and extracted the dispersion relation for kaonic excitations. There appear two modes: Goldstone-like soft mode and very massive one, which correspond to $K^{-}$and $K^{+}$ mesonic excitations, respectively. We have discussed that the form of the dispersion relation can be reduced to a simple one by the approximation, $c_{3}=0$, which was also checked numerically and proved to be fairly good.

Applying our formalism to the derivation of EOS as an example, we have pointed out the largeness of the thermal effects in the condensed phase; they result in a remarkable reduction of pressure in the condensed phase, compared with the normal phase.

In this Letter we have not taken into account the dynamical degrees of freedom of nucleons consistently, but it is not difficult to treat them consistently based on the idea given in this Letter [14].

We thank T. Muto for discussions and careful reading of this manuscript. This work was supported in part by the Japanese Grant-in-Aid for Scientific Research Fund of the Ministry of Education, Science and Culture (08640369). 


\section{Figure Captions}

Fig. 1 Dispersion relation for kaonic modes in the condensed phase at $u=3.87$ and $T=50$ $\mathrm{MeV}$. Solid lines show the results given by Eq. (19), while dotted lines by Eq. (18).

Fig. 2 Equation of state for neutron-star matter at finite temperature. EOS at $\mathrm{T}=0$, which is the same one given in ref. [5], is also shown for comparison. Dotted lines show EOS for normal neutron-star matter. 


\section{REFERENCES}

[1] D.B. Kaplan and A.E. Nelson, Phys. Lett. B175 (1986) 57 ; B179 (1986) 409(E).

For a recent review, C.-H. Lee, Phys. Reports 275 (1996) 197.

[2] G.E. Brown and H.A. Bethe, Astrophys. J. 423 (1994) 659.

[3] T.W. Baumgarte, S.L. Shapiro and S. Teukolsky, Astrophys. J. 443 (1995) 717; 458 (1996) 680.

[4] H. Fujii, T. Maruyama, T. Muto and T. Tatsumi, Nucl. Phys. A597, 645 (1996). As a review, T. Tatsumi, Prog. Theor. Phys. Suppl. 120 (1995) 111 and references cited therein.

[5] V. Thorsson, M. Prakash and J.M. Lattimer, Nucl. Phys. A572 (1994) 693; A574 (1994) 851.

[6] W. Keil and H.-Th. Janka, Astron. Astrophys. 296 (1995) 145.

[7] M. Prakash, I. Bombaci, M. Prakash, P.J.Ellis, J.M. Lattimer, R. Knorren, Phys. Reports 280 (1997) 1.

[8] V. Thorsson and P.J. Ellis, Phys. Rev. D55 (1997) 5177.

[9] E. Jenkins and A. Manohar, Nucl. Phys. B368 (1992) 190.

[10] H. Georgi, Weak interactions and modern particle theory (Benjamin/Cummings Pub., 1984).

[11] S. Weinberg, Phys. Rev. 166 (1968) 1568; Physica 96A (1979) 327; The quantum theory of field II (Cambridge U. Press, 1996).

[12] J. Gasser and H. Leutwyler, Phys. Lett. B188 (1987) 477.

F.C. Hansen, Nucl. Phys. B345 (1990) 685.

[13] M. Prakash, T.L. Ainsworth and J.M. Lattimer, Phys Rev. Lett. 61 (1988) 2518.

[14] T. Tatsumi and M. Yasuhira, in preparation. 